\title{
The Design of a Motion Control System for a Parallel Robot with Image Positioning
}

\author{
Chao-Shu Liu ${ }^{1}$, Wei-Lun Chang ${ }^{2}$ and Chi-Hao Lin ${ }^{2}$ \\ 1. Department of Mechanical Engineering, National Kaohsiung University of Applied Sciences, Kaohsiung City 807, Taiwan, R.O.C \\ 2. Institute of Mechanical and Precision Engineering, National Kaohsiung University of Applied Sciences, Kaohsiung City 807,
}

Taiwan, R.O.C.

\begin{abstract}
A motion control system for a parallel robot with image positioning was implemented in this paper. The system is composed of a machine vision device, a delta robot and a linear stage, and the concerned hardware, software and working methods were developed completely and verified successfully. During the phase of machine vision, the image of object was captured by camera, and then the process of smoothing filter, threshold algorithm and edge detection, was applied so as to obtain the edges of image. Finally, DV-GHT (Displacement Vector Generalized Hough Transformation) algorithm was used to recognize the center of multiple and arbitrary 2-D shapes objects. After the center of objects was recognized, the objects were delivered to the workspace of a delta robot by a motorized stage. Through the coordinate transformation between the camera and the robot system, the information of center can be converted to control commands for every working motors. Following, the delta robot picks up objects to the specified position sequentially by the trajectory planning and tracking controls. The software of $\mathrm{C}++/ \mathrm{CLI}$ is used to achieve the phase of motion controls and the program of DV-GHT is used to detect and conduct the positions for four different characteristics of the objects simultaneously so as to indicate the delta robot to classify the objects successfully.
\end{abstract}

Key words: DV-GHT, machine vision, Delta robot.

\section{Introduction}

Concerning the applications of parallel robots with machine vision, parts aligning and object packaging are often applied. But it is seldom to find the system that can work simultaneously for various kinds of parts. Therefore, the objective of this paper is to combine the technologies of machine vision and image recognition with the researches of reference [1], the motion controls of a parallel robot, so as to construct an integrated automation system composed of machine vision, a parallel robot and an electrical linear stage. After the image of objects are captured, the positions of these objects would be recognized by the methods of image processing, then these objects would be picked up and placed into different stations sequentially through the trajectories programming and robot

Corresponding author: Chao-Shu Liu, Ph.D., associate professor, research fields: system integration, and robotic design and control. controls. An automatic classifying system for multiple and arbitrary 2-D shapes objects would be achieved finally.

About the image positioning, the position information is needed for a robot to arrest objects. In 1962, P. Hough addressed a method of HT (Hough transformation) that could map an image space into a parameter space so as to form an accumulation table and the shape parameter is the biggest value of the accumulation table. However, the calculation time would be wasted much and the larger capacity of memory would be needed due to the increasing of the parameter space dimensions. Therefore, many improving methods for calculation were provided, such as Fast HT addressed by H. Li et al. [12] and Random HT proposed by Lex XU et al. [13]. But they are adopted for the model that can be parameterised only, such as the shapes of linear, circle, ellipse, etc.

The characteristics of different objects need to be 
observed to find an appropriate algorithm. However, it is hard to find the corresponding equations for all shapes. Therefore, Ballad [14] provided the method of GHT (generalized Hough transformation) in 1981 to construct an $\mathrm{R}$ table through the vector information of reference points and edge points, and the gradient information of edge points, and then applying the $\mathrm{R}$ table to detect the graph. According to the calculation of possible positions of the shapes and the accumulation of the values in Hough Space, the peak value of the parameters was the position of the detected shape. The Hough Space would be extended from 2-D space to 4-D space if considering the parameters of stretch and rotation so as that the calculation time and memory space would be wasted very much. Therefore, many methods to improve the efficiency of calculation were provided later.

D. Ma et al. [15] provided a method, SC-GHT, to calculate the parameters of stretch and rotation by using slope and curvature, but it is ill-fitting to the object featured by linear line. H. M. Lee et al. [16] provided a method, ER-GHT, to reduce 2-D space to 1-D by considering the edge points of an object to be reference point, but it is ill to calculate the position for the image with broken edge points. Ser and Siu [17] provided a pair of edge points to construct R table, RG-GHT, but it is unavailable to the object that is masked or has broken edge points. Thomas [18] proposed a displacement vector to index R Table. Kassim et al. [19] modified the method and provided DV-GHT to calculate the values of position, ratio and rotation simultaneously by the accumulation table in a 3-D space. The method does not waste the memory space too much to construct the accumulation table and has good calculation efficiency, so the method, DV-GHT, to be the positioning algorithm, was applied in this paper.

\section{Image Positioning}

\subsection{DV-GHT (Displacement Vector Generalised Hough Transformation)}

It is very complex for typical Hough Transformation to consider the parameters of position, ratio and rotation when detecting ratio and rotation. Therefore, the paper would apply the method, DV-GHT, to overcome the problem. The method uses a displacement vector to index $\mathrm{R}$ table so as to reduce the complexity of space and time, and to calculate the parameters of position, ratio and rotation simultaneously in 3-D Hough Space.

The process of detection can be divided into 2 phases:

(a) R-Table Construction Phase

DV-GHT uses $\mathrm{R}$ table to record the vector information, from every edge point to reference point, the gradient information for every edge point, and gradient orientation $\Phi$.

In Fig. 1, firstly a reference point $\left(x_{r}, y_{r}\right)$ is selected, generally it is the position of shape center. Then calculating the values of vector, orientation, $(r, \alpha)$ and gradient orientation $\Phi$ for every edge point $(x, y)$. The angle error vector, $e=\Phi-\alpha$, between the vector of gradient orientation and the vector of edge point to reference point, can be recorded by index $(r, \alpha, \Phi)$ for all edge points so as to construct the $R$ table, the data format are shown in Fig. 2.

$$
\begin{gathered}
r=\sqrt{\left(x-x_{r}\right)^{2}+\left(y-y_{r}\right)^{2}} \\
\alpha=\tan ^{-1}\left(\frac{y-y_{r}}{x-x_{r}}\right)
\end{gathered}
$$

(b) Shape Detection Phase

Firstly initializing a 3-D Hough $\operatorname{Space} \operatorname{Acc}\left(x_{c}, y_{c}, s\right)$, then calculating the gradient orientation $\Phi$ for every

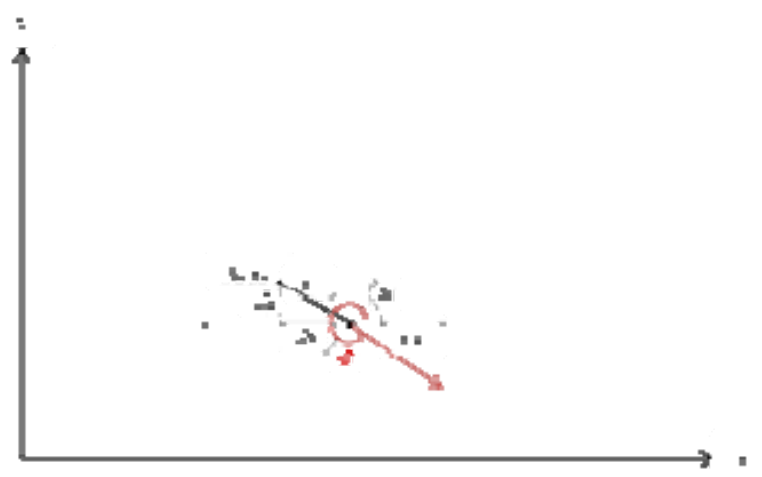

Fig. 1 DV-GHT for arbitrary shape. 


\begin{tabular}{|c|c|}
\hline index & entries \\
\hline$e_{0}$ & $\left(r_{1}, \alpha_{1}, \Phi_{1}\right)_{0} \cdot\left(r_{2}, \alpha_{2}, \Phi_{2}\right)_{\theta} \ldots \ldots\left(r_{m}, \alpha_{m}, \Phi_{m}\right)_{a}$ \\
\hline$e_{1}$ & $\left(r_{1}, \alpha_{f}, \Phi_{1}\right)_{1} \cdot\left(r_{2}, \alpha_{2}, \Phi_{2}\right)_{1} \ldots \ldots\left(r_{m}, \alpha_{m}, \Phi_{m}\right)$, \\
\hline$e_{2}$ & $\left(r_{1}, \alpha_{1}, \Phi_{t}\right)_{2} \cdot\left(r_{2}, \alpha_{2}, \Phi_{2}\right)_{2} \ldots \ldots\left(r_{m}, \alpha_{m}, \Phi_{m}\right)_{2}$ \\
\hline$\vdots$ & $\vdots$ \\
$\vdots$ & $\vdots$ \\
- & $\vdots$ \\
\hline$e_{n}$ & $\left(r_{t}, \alpha_{t}, \Phi_{t}\right)_{n} \cdot\left(r_{2}, \alpha_{2}, \Phi_{2}\right)_{n} \ldots \ldots\left(r_{m}, \alpha_{m}, \Phi_{m}\right)_{n}$ \\
\hline
\end{tabular}

Fig. 2 R-table data format.

edge point and all of indices $\Phi_{m}$. Let $\Phi_{e}=\Phi-\Phi_{m}$ and $\left(x_{c}, y_{c}\right)$ can be calculated and accumulated in an accumulation table $\operatorname{Acc}\left(x_{c}, y_{c}, s\right)$. Finally the peak value can be found to be the shape parameter.

$$
\left[\begin{array}{l}
x_{c} \\
y_{c}
\end{array}\right]=\left[\begin{array}{l}
x \\
y
\end{array}\right]+s r\left[\begin{array}{c}
\cos (\alpha+\theta) \\
\sin (\alpha+\theta)
\end{array}\right]
$$

For example, there are many detected objects shown in Fig. 3. Four objects are selected to construct R table shown in Fig. 4. Fig. 5 is the edge detection results for Fig. 3. The results of DV-GHT are shown in Fig. 6.

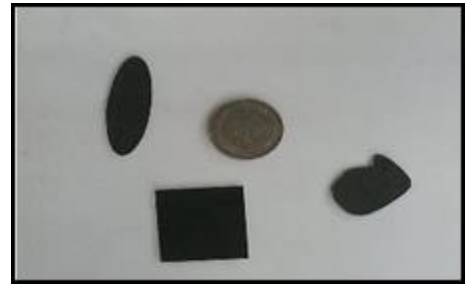

Fig. 3 Original image.



Fig. 4 Patterns.

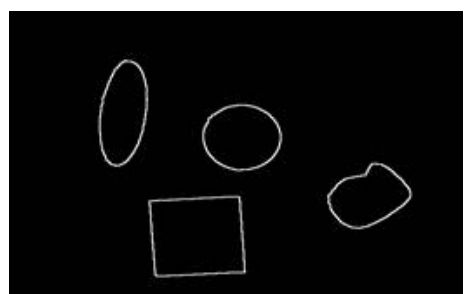

Fig. 5 The edge detection results.

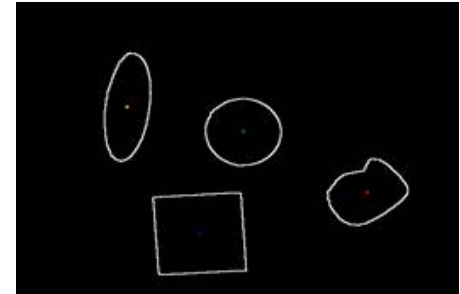

Fig. 6 The results of DV-GHT.

\subsection{The Process of Image Positioning}

About the image positioning, the process is listed in Fig. 7. In order to get the image with complete edges, the original image must be greyed and the noise must be attenuated by mean value filter. Then using the method, adaptive threshold value, to separate the foreground and backgroud for an image with unbalanced illumination. When the foreground is obtained, the method of close morphology is applied to fill the small holes and connect the broken lines. If the method cannot fill the inner holes, the method of flood filling is used to fill the holes, and then detecting the image edge by the method of Sobel edge detection. Finally, the coordinates of the detected objects can be gotten by the algorithm of DV-GHT.



Fig. 7 The process of image positioning. 
When the robot works to do the classification operation, the coordinates of the objects must be needed. Therefore, it is essential to translate the image coordinate into the robot coordinate system that includes static and dynamic coordinate translations.

In Fig. 8, the origin of the robot coordinate system is $O$, the end point of the cupule is $P$, the bench center of the linear stage is $O_{m}$ and the origin of the camera coordinate system is $O_{c}$.

(a) Coordinate Translation for Static Objects

Coordinate translation for static objects is assumed under the condition that the working bench equipped on the linear stage stops moving when it is in the working area of the robot, then the robot begins to pick up the objects and put them to classification positions sequentially.

When $O_{m}$ is under $O_{c}$, the image recognition is executed. After the recognition propcess, the working bench is moved to $O_{m}$ ' and then stopped. Because the distance between $P$ and $O_{m}$ ', and the angle $\theta$ between these coordinate systems have been known, the coordinate information for the robot can be obtained through the matrices of rotation and translation. In which $[x, y, z]$ is the coordinate of the object in camera coordinate system, $\left[x_{s}, y_{s}, z_{s}\right]$ is the coordinate of the object in robot coordinate system.

(b) Coordinate Translation for Dynamic Objects

Coordinate translation for dynamic objects is under the condition that the working bench equipped on the linear stage continues moving in a constant speed and the robot picks up the objects and puts them to classification positions.

In the paper, the linear stage is equipped along the direction of $x$, so the objects are moving in the same dircetion and their positions are estimated as the change of $x$ coordinate only. In Fig. 9, the distance between $O_{c}$ and $O$ is $S$ in the directoin of $X . S(t)$ is the position of working bench due to $\mathrm{O}_{\mathrm{c}}$ for every instant $t$. Therefore, the dynamic coordinate $\left[x_{d}, y_{d}, z_{d}\right]$ can be calculated for the robot system, in which $\left[x_{s}, y_{s}, z_{s}\right]$ is the stastic translation when $d x=0$. Finally the distance $S(t)$ can be

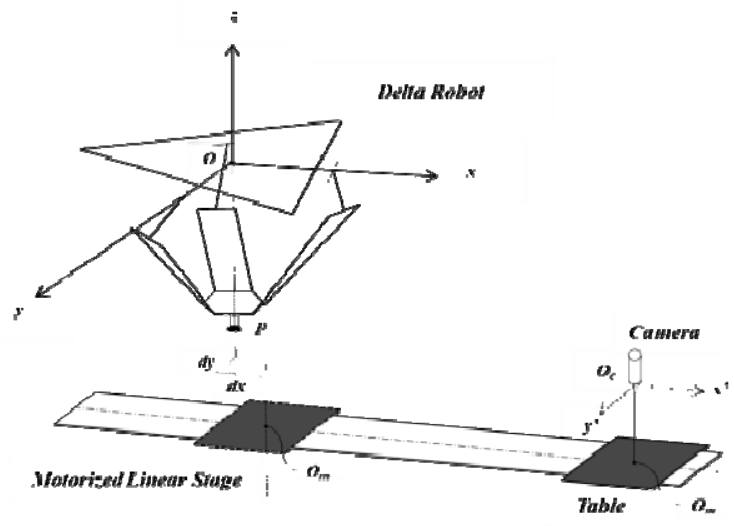

Fig. 8 The coordinate systems of robot and image.



Fig. 9 The distance estimation for moving objects.

obtained for the working bench in the constant speed $v_{m}$.

$$
S(t)=v_{m} \cdot t
$$

\section{Experimental Results}

The experimental bench is composed of a parallel robot, an electrical linear stage and machine vision, and is shown in Fig. 10. The hardware structure includes a PC, a digital I/O card, CMOS camera, a parallel robot, 3 axes servo motor motion control board, electrical linear stage and a stepping motor control board.

The experimental bench has two working stations as Fig. 11. The first station is the area of vision recognition and the second station is the working area for robot control system. The objects are moved to make vision recognition in the first station, and then moved to the second station, finally the parallel robot is controlled to pick up the objects and make classification. 




Fig. 10 The hardware structure.

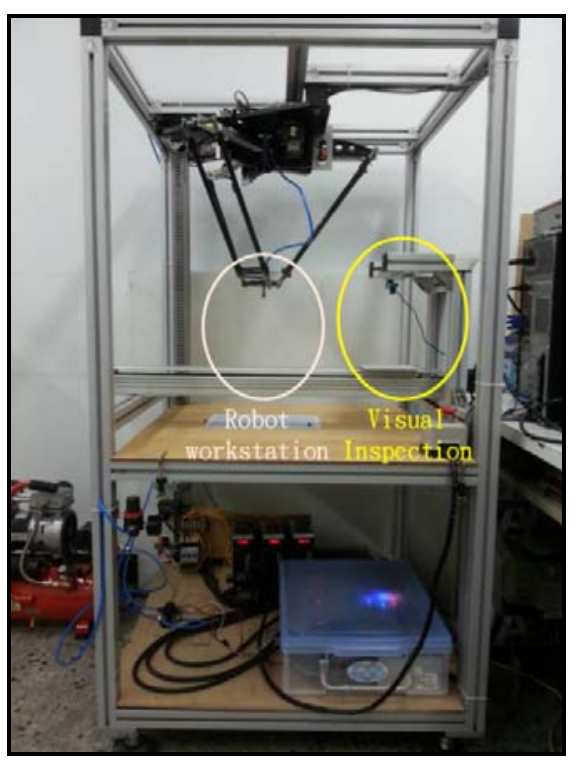

Fig. 11 The experimental bench.

\subsection{DV-GHT Positioning Precision Experiments}

In the section, the experiemnts do not consider the parameter of ratio, so Hough Space is 2-D and different $\mathrm{R}$ table is used to record information for different ratio objects.

\subsubsection{Positioning Experiments for an Ideal Image}

There are four different shapes drawn on the set coordinates in a $640 \times 480$ image in advance as Fig. 12. Then observing the position bias, in which the origin $(0,0)$ of the image coordinate is set on the corner of the left up side, and four $\mathrm{R}$ tables are used to construct the information for four different objects. The positioning results for various objects are shown in Fig. 13 and Table 1, and the positioning error is \pm 1 pixel.

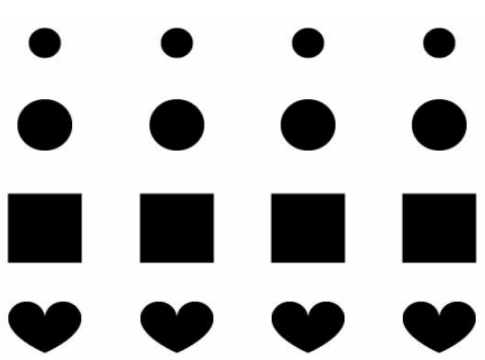

Fig. 12 Different shapes in a $640 \times 480$ Image.

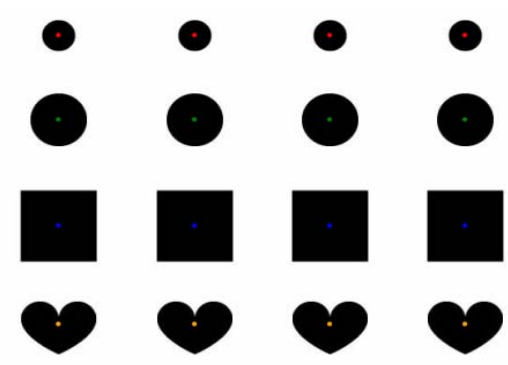

Fig. 13 The positioning results.

Table 1 DV-GHT for various objects.

\begin{tabular}{ll}
\hline Known coordinates (pixel) & $\begin{array}{l}\text { Positioning coordinates } \\
\text { (pixel) }\end{array}$ \\
\hline$(103,66)$ & $(102,65)$ \\
$(248,66)$ & $(247,65)$ \\
$(392,66)$ & $(391,65)$ \\
$(536,66)$ & $(535,65)$ \\
$(103,162)$ & $(102,161)$ \\
$(248,162)$ & $(247,161)$ \\
$(392,162)$ & $(391,161)$ \\
$(536,162)$ & $(535,161)$ \\
$(103,283)$ & $(102,282)$ \\
$(248,283)$ & $(247,282)$ \\
$(392,283)$ & $(391,282)$ \\
$(536,283)$ & $(535,282)$ \\
$(103,395)$ & $(102,394)$ \\
$(248,395)$ & $(247,394)$ \\
$(392,395)$ & $(391,394)$ \\
$(536,395)$ & $(535,394)$ \\
\hline
\end{tabular}

3.1.2 Positioning Experiments for Camera Image

The real image is captured by camera with the resolution of $640 \times 480$ pixeles. For practical applications, the origin of the image coordinate would be set as $(320,240)$, in which 1 pixel $=0.1828125 \mathrm{~mm}$.

There are 12 calibration pictures with known center coordinates put under the camera as Fig. 14 and then the bias can be observed. Finally the positioning 


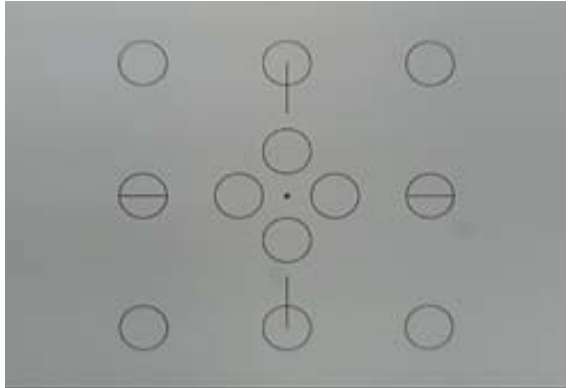

Fig. 14 Twelve calibration pictures.

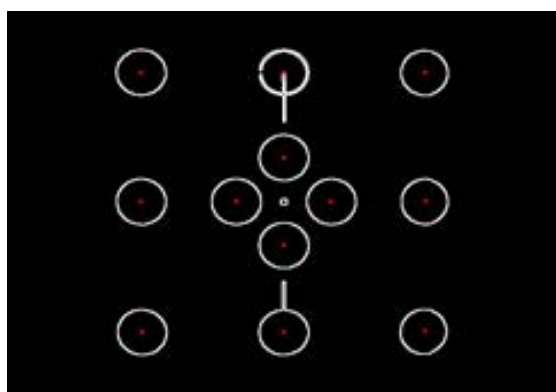

Fig. 15 The positioning results.

Table 2 Positioning results for calibration pictures.

\begin{tabular}{ll}
\hline Known coordinates $(\mathrm{mm})$ & Positioning coordinates $(\mathrm{mm})$ \\
\hline$(-30,30)$ & $(-29.7984,29.7984)$ \\
$(0,30)$ & $(0,29.7984)$ \\
$(30,30)$ & $(29.6156,29.6156)$ \\
$(-30,0)$ & $(-29.9813,0)$ \\
$(30,0)$ & $(29.79844,0)$ \\
$(-30,-30)$ & $(-29.9813,-29.9813)$ \\
$(0,-30)$ & $(0,-29.9813)$ \\
$(30,-30)$ & $(29.6156,-29.9813)$ \\
$(0,10)$ & $(0,9.8719)$ \\
$(-10,0)$ & $(-9.8719,0)$ \\
$(10,0)$ & $(10.0547,0)$ \\
$(0,-10)$ & $(0,-10.0547)$ \\
\hline
\end{tabular}

results are shown in Fig. 15 and Table 2, and the positioning error is about $\pm 0.4 \mathrm{~mm}$.

\subsection{Trajectory Tracking Experiments}

The experiments are the trajectory tracking controls for a parallel robot and the control parameters are listed in Table 3. The control period is $500 \mathrm{~ms}$.

The control command is the motion on $x-y$ plane and the control results are shown in Table 4. After calculating the distance, the trajectory tracking error is $0.1 \mathrm{~mm}$. Observing Figs. 16-18, the control curves are smoother than point-to-pint controls and the error
Table 3 The control parameters.

\begin{tabular}{ll}
\hline Control parameters & \\
\hline Motion period (s) & 0.5 \\
Sampling rate (Hz) & 1,000 \\
MDDS parameters & $c_{1}=c_{2}=c_{3}=50$ \\
Initial point $(\mathrm{mm})$ & {$[0,0,-334.03]$} \\
Target point $(\mathrm{mm})$ & {$[50,50,-334.03]$} \\
\hline
\end{tabular}

Table 4 The control results.

\begin{tabular}{ll}
\hline Control results \\
$\begin{array}{l}\text { The position after control } \\
(\mathrm{mm})\end{array}$ & {$[49.9535,49.9338,-334.0301]$} \\
Positioning error $(\mathrm{mm})$ & 0.0809 \\
\hline
\end{tabular}


Fig. 16 Trajectory tracking control-position response.
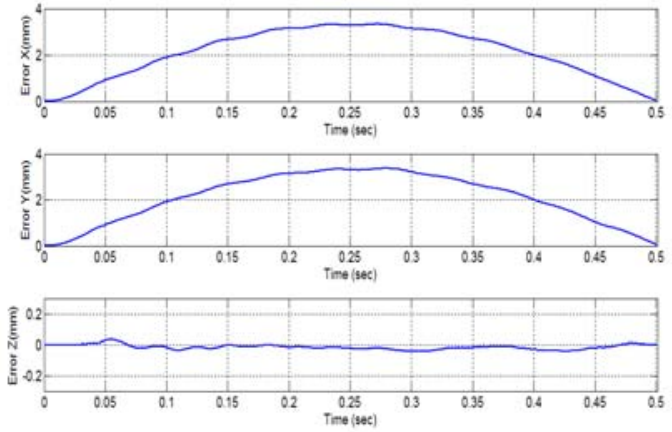

Fig. 17 Trajectory tracking control-position error.
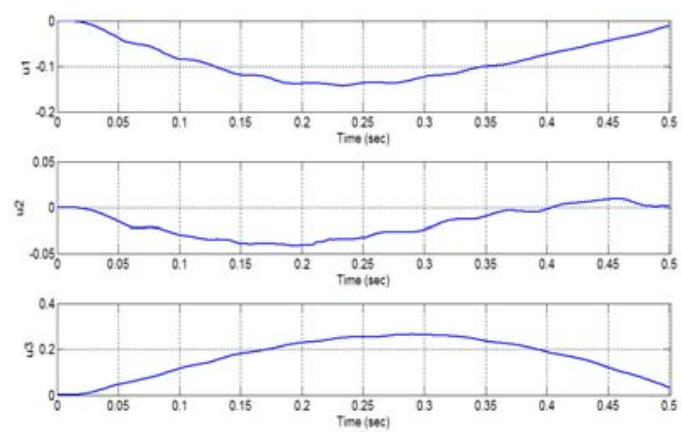

Fig. 18 Trajectory tracking control—control effort. 


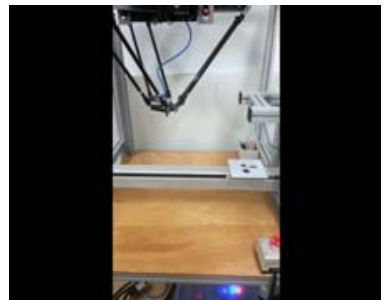

(1)



(3)

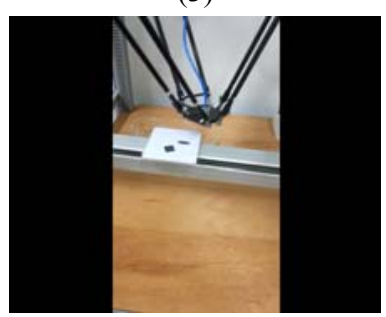

(5)

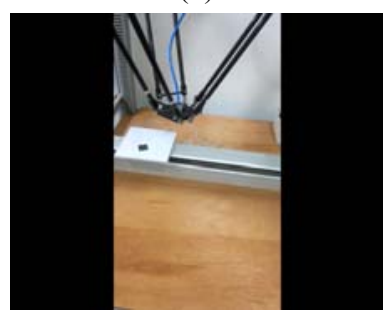

(7)



(9)

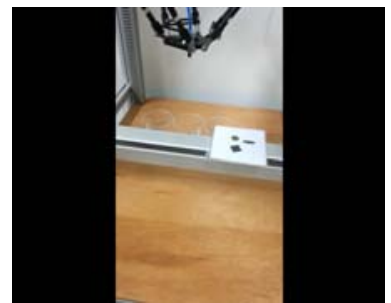

(2)

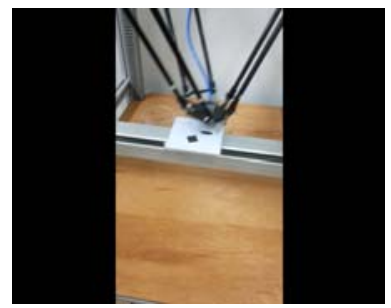

(4)



(6)

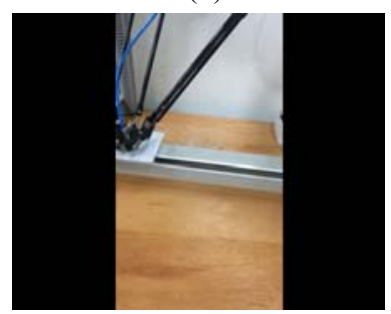

(8)

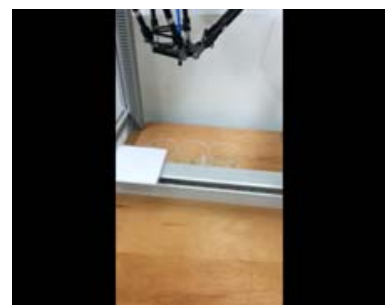

(10)
Fig. 19 The process of objects classification: (1)-(10).

is $\pm 0.1 \mathrm{~mm}$ in $\mathrm{z}$ direction. Finally the process of objects classification is shown in Fig. 19.

\section{Conclusions}

The integrated automation system of a parallel robot and machine vision was constructed in this paper. An image processing algorithm was applied in the system to make the working robot execute positioning and trajactory tracking controls through the information captured by camera so as to run classification operation. In order to detect the objects with arbitrary shapes, the method, DV-GHT, was applied to be the positioning algorithm. The implementation of motion control and image positioning was designed by the program language C++/CLI. From the experimental results, the positioning error for DV-GHT is within \pm 1 pixel and the trajectory tracking error is within $\pm 0.1 \mathrm{~mm}$.

The design can classify four kinds of objects simultaneously and finally the hardware and software for the integrated system of machine vision and a parallel robot are finished in the paper successfully.

\section{References}

[1] Lin, H.-H., Tai, Y.-H., and Liu, C.-S. 2013. "The Implementation of Smoothing Robust Control for a Delta Robot." The Second International Conference on Robot, Vision and Signal Processing, RVSP2013-IS12-0009.

[2] Shirai, Y., and Inoue, H. 1973. "Guiding a Robot by Visual Feedback in Assembling Tasks." Pattern Recognition 5: 99-108.

[3] Hill, J., and Park, W. T. 1979. "Real Time Control of a Robot with a Mobile Camera." In Proceedings of the 9th ISIR, 233-46.

[4] Doyle, W. 1962. "Operation Useful for Similarity-Invariant Pattern Recognition.” J. Assoc. Comput. Machinery 9: 259-67.

[5] Kapur, J. N., Sahoo, P. K., and Wong, A. K. C. 1985. “A New Method for Gray-Level Picture Thresholding Using the Entropy of the Histogram." Computer Vision, Graphics and Image Processing 29: 273-85.

[6] Nobuyuki, O. 1979. "A Threshold Selection Method from Gray-Level Histograms.” IEEE Transactions on Systems, Man, and Cybernetics, SMC-9, 1: 62-4.

[7] Huang, Q., Gao, W., and Cai, W. 2005. "Thresholding Technique with Adaptive Window Selection for Uneven Lighting Image." Pattern Recognition Letters 26: 801-8.

[8] Pradhan, S. S., Patra, D., and Nanda, P. K. 2008. "Adaptive Thresholding Based Image Segmentation with Uneven Lighting Condition." Proc. on IEEE Region 10 Colloquium and the Third ICIIS.

[9] Wellner, P. D. 1993. Adaptive Thresholding for the Digital Desk. Tech. Rep. EPC-1993-110.

[10] Bradley, D., and Roth, G. 2007. "Adaptive Thresholding Using Integral Image.” Graphics Tools 12: 13-21.

[11] Hough, P. V. C. 1962. "Method and Means for 
Recognizing Complex Patterns." U.S. Patent No. 3069654.

[12] Li, H., Lavin, M. A., and Le Master, R. J. 1986. "Fast Hough Transform: A Hierarchical Approach." Computer Vision, Graphics and Image Processing 36: 139-61.

[13] Xu, L., Oja, E., and Kultanen, P. 1990. “A New Curve Detection Method: Randomized Hough Transform (RHT)." Pattern Recognition Letters 11: 331-8.

[14] Ballard, D. H. 1981. "Generalized the Hough Transform to Detect Arbitrary Shapes." Pattern Recognition 3: 110-22.

[15] Ma, D., and Chen, X. 1988. "Hough Transform Using Slope and Curvature as Local Properties to Detect Arbitrary 2D Shapes." In Proc. 9th Int. Conf. on Pattern Recognition: 511-3.

[16] Lee, H. M., Kittler, J., and Wong, K. C. 1992. "Generalised Hough Transform in Object Recognition." Proc. 11th IAPR International Conference on Pattern
Recognition, IEEE Computer Society Press 3: 285-9.

[17] Ser, P.-K., and Siu, W.-C. 1994. "Non-analytic Object Recognition Using the Hough Transform with Matching Technique." IEE Proc-Comput. Digit. Tech. 141: 11-6.

[18] Thomas, A. D. H. 1993. "Compressing the Parameter Space of the Generalized Hough Transform." Pattern Recognition Letters 13: 107-12.

[19] Kassim, A. A., Tan, T., and Tan, K. H. 1999. "A Comparative Study of Efficient Generalised Hough Transform Techniques." Image Vision Computing 17: 737-48.

[20] Suh, S.-B. 2008. "Computing State Migration between Mobile Platforms for Seamless Computing Environments." IEEE CCNC: 1216-7.

[21] Nunnally, P. C. 1989. "An Inexpensive Robot Arm for Computer Vision Applications." Southeastcon '89. Proceedings. Energy and Information Technologies in the Southeast, IEEE 1: 1-6. 